\title{
ANALYSIS OF TECHNICAL CRITICALITIES FOR GIS MODELLING AN URBAN NOISE MAP
}

\author{
Gino DARDANELLI ${ }^{1}$, Rosario MARRETTA ${ }^{2}$, Antonio Sansone SANTAMARIA ${ }^{2}$, \\ Antonino STREVA ${ }^{2}$, Mauro LO BRUTTO ${ }^{1}$, Antonino MALTESE ${ }^{1}$
}

DOI: $10.21163 / G T \_2017.122 .05$

\begin{abstract}
:
This paper analyzes criticalities and strengthens of a procedure used to model the acoustic map of the vehicular traffic of an urban agglomeration. The research is based on a pilot project for the acoustic mapping of a portion of the city of Palermo (Italy). Simulations indicate that the acoustic climate was in line with expectations and with typical of large Italian cities. The most remarkable result was obtained by an increase in the number of reflections (from two to five), while the influence of the height of the building (from 9 to 18 meters) was negligible - on the order of a few points per thousand. Regarding the analysis conducted with the "Gden Method", acoustic values do not diverge significantly from the other Italian cities, registering values that were, however, the highest in the investigated sample.

Key-words: GIS, noise map, acoustic models, ICT, END, Gden method.
\end{abstract}

\section{INTRODUCTION}

Today, in European Union countries, noise pollution is one of the biggest environmental problems of city life and is certainly one of the most significant causes of the decrease in the quality of the life. In recent decades, this acoustic phenomenon and its attendant problems have become increasingly worrying, not just in densely populated urban environments with elevated traffic flows, but also in special infrastructure (airports, racetracks, industrial areas) whose activities naturally produce high levels of sound pressure. Particularly, in the last years, many studies have shown that some of the most critical noise sources in our environment are associated with transport (Prekop and Dolejšs, 2016, Covaciu et al. 2010). In order to address this issue and to protect human health and well-being, a legislative framework has been established in Italy for years (Sicily Region Office, 2017). It provides a classification of the territory into areas within which certain day and night noise limits should not be exceeded. The law is structured to provide limits for each of the noise indicators. Indicators must not be exceeded, according to the exposure class and the reference period; the law also recommends supervisory actions and remedial measures to be taken when the limits are exceeded. Today, obligations established by national legislation have been only partially implemented, and there are considerable differences between the different areas and between the different fields covered by national

\footnotetext{
${ }^{1}$ University of Palermo, DICAM, 90128 Palermo, Italy, gino.dardanelli@ unipa.it; mauro.lobrutto@unipa.it; antonino.maltese@unipa.it

${ }^{2}$ Laboratory of physical agents of ARPA SICILIA, 90129 Palermo, Italy, asansone@arpa.sicilia.it; rmarretta@arpa.sicilia.it; ing.antonino.streva@gmail.com
} 
law (Curcuruto et al. 2012). A new strategy to challenge the problem of environmental noise is provided by European Environmental Noise Directive (END) that introduced two new describers to create acoustic maps in urban agglomerations, so as to highlight zones where certain fixed limits are exceeded (Murphy and King, 2010). To comply with the Directive, Member States are required to produce strategic noise maps of designated areas that characterize noise produced by the principal roads. These maps must employ European indicators $\mathrm{L}_{\mathrm{den}}$ and $\mathrm{L}_{\text {night }}$ (O’Malley et al. 2009). As for the obligations established by END, here, too, there are significant delays in Italy in the assignment of the works and the information furnished is often incomplete. Elsewhere in Europe, however, there is a high level of attention to the issue and to the deadlines imposed by the laws. On the other hand, long delays are found in the search for solutions to noise problems in the United States (Seong et al. 2011), South America (Bastián-Monarca et al. 2016) and China (Cai et al. 2015), which only recently have begun to find remedies to the phenomenon. Returning to the European context, there are cases of noise maps realized even before of the publication of END. In 1998, Birmingham, United Kingdom, was one of the first cities to produce a detailed acoustic map and Linz, Austria, followed, where the urgency was to the need to address the problems created by the heavy industry in urban areas (Knauss, 2002). The Paris noise map has existed since 2001. It was an imposing job (six thousand streets with about 1.2 million vehicles a day) completed after years of measurements and calculations (Hepworth, 2006).

To create acoustic maps, it is first essential to collect suitable and complete data. In addition, these data must be in a format that allows for their immediate interpretation and fruition. In this context, it is, therefore, essential to create and use databases and information systems that are complete and at the same time sufficiently dynamic to follow changes in the factors involved both in the short and the long term. The second point in realizing a thematic map is the suitability of the cartographic base. The completeness, clarity and accuracy of numerical maps simplify the exchange and integration of the information contained in databases, thereby guaranteeing the correct placement of each entity. A preliminary study of acoustic mapping by vehicular traffic was carried out in Palermo in last years (Dardanelli et. al 2010).

The objective of this research is the analysis of technical criticalities for GIS (Geographical Information System) modelling an urban noise map to each noise band obtained from a simulation study, as well as the acoustic mapping of a large urban city. The work was carried out by employing open source data. An insufficient amount of data was previously collected, that were unsuitable for implementing an appropriate analysis under current Italian law. At the same time, to realize the acoustic map, the procedure should ensure the measurement of the estimated sound levels (geo-referencing of data) within the selected territory by means of an automatic procedure, or else a manual procedure, on a GIS. In light of this, a large part of the job concerned the study of a methodology that by using available databases (cartographic tools and measurements) could obtain georeferenced information that covered large parts of the urban fabric. The results achieved can be considered a starting point to create GIS that is well-constructed, complex and easily 
integrated, and, at the same time, aimed at an analysis of the noise problem in cities. Meanwhile, the GIS must form the basis for a correct weighing of the different environmental issues.

\section{THEORY AND METHOD}

The cartographic maps, the available databases and various operational choices made in during the realization of the simulation allowed assessing the adequacy of the data and, at the same time, to estimate the weight that certain geometrical parameters exercise on the acoustic map - one of them is the number of reflections on facades. Although the steps taken seem to have been executed sequentially and without complications, various issues arisen often obliged us to go back to correct or add information to the operations performed previously. Principally, this was because the information was neither complete nor updated and also because this information was very often in an unsuitable format to rapid implementation.

In this somewhat iterative process, however, it is possible to distinguish the following steps:

- analysis of cartography, specifically regarding the morphology of the land, and then the creation of a Digital Elevation Model (DEM);

- integration of cartographic map with information on the resident population in the area;

- analysis and integration of traffic flows and creation of a database of noise sources;

- realization and analysis of acoustic maps.

The software used included an open source GIS (Quantum GIS 2.6.1 'Nødebo'), a noise simulator software (SoundPLAN 7.3 by Spectra s.r.1.) and ViaTerm and ViaGraph software for processing the data obtained from equipment to a count traffic device (Viacount II), developed by Via traffic controlling.

The following quantity is defined as sound pressure level $(\mathrm{dB})$ :

$$
L_{p}=10 \log \left(\frac{p}{p_{0}}\right)^{2},
$$

Where:

- $\mathrm{p}$ is the root mean square of the sound pressure $(\mathrm{Pa})$;

- $\mathrm{p}_{0}$ is the reference sound pressure. The commonly used reference for sound pressure in air is $2 * 10-5 \mathrm{~Pa}$.

To describe environmental noise in a comprehensive way, changes over time must be taken into account; this stems from the fact that environmental noise fluctuates among values varying over time. For this reason, it is necessary to introduce the Equivalent Continuous A-Weighted Sound Pressure Level, LAeq:

$$
L_{A \varepsilon q, T}=10 \log \left[\frac{1}{T} \int_{0}^{T} \frac{p_{A}^{2}(t)}{p_{0}^{2}} d t\right],
$$

Where:

- $\mathrm{p}_{\mathrm{A}}(\mathrm{t})$ is the instantaneous A-weighted sound pressure $(\mathrm{Pa})$ of the sound signal; 
- $\mathrm{T}$ is the integration time interval (s);

- $\mathrm{L}_{\text {Aeq, } \mathrm{T}}$ is the average energy level expressed in $\mathrm{dB}(\mathrm{A})$.

The noise indicators used to develop and update acoustic maps are Lden (day, evening and night) and Lnight (night). The day-evening-night level Lden in decibels (dB) is defined by:

$$
L_{d e n}=10 \log \frac{1}{24}\left(t_{d} * 10^{\frac{L_{d a y}}{10}}+t_{e} * 10^{\frac{L_{\text {avening }}+5}{10}}+t_{\mathrm{n}} * 10^{\frac{L_{\text {night }}+10}{10}}\right),
$$

Where:

$-\mathrm{L}_{\text {day }}$ is the A-weighted long-term average sound level as defined in ISO 1996-2: 1987, determined over all the day periods of a year;

- $\mathrm{L}_{\text {evening }}$ is the A-weighted long-term average sound level as defined in ISO 1996-2: 1987, determined over all the evening periods of a year;

- $\mathrm{L}_{\text {night }}$ is the A-weighted long-term average sound level as defined in ISO 1996-2: 1987, determined over all the night periods of a year;

$-t_{d}$, te and th represent the period expressed in hours.

The day is assumed to last 12 hours, the evening 4 hours and the night 8 hours. The Member States may shorten the evening period by one or two hours and lengthen the daytime and/or the nighttime period accordingly, provided that this choice is applied for all sources and that they provide the Commission with information on any systematic difference from the default option. In some cases, in addition to $\mathrm{L}_{\mathrm{den}}$ and $\mathrm{L}_{\text {night }}$, it may be useful to use supplementary noise indicators to deal with particular circumstances in which the measurements are taken. However, all descriptors should be assessed by using the measurement and calculation methods set out in Annex II of END. The Directive also recommends provisional methods of calculation for the Member States that don't have their own national method.

Neglecting other sources of noise, as they are not within the scope of this job, the official method for the calculation of vehicular noise is indicated out as the French method. This is the "Nouvelle Methode de Prevision de Bruit" (NMPB - Routes-96) developed by French Institutes that constitute the Technical Services of the Ministère de L'Equipement (SETRA-CERTU-LCPC-CSTB, 1997). The method, aimed exclusively at the modeling of road traffic noise, evolved from a method used in the 1980s. This development became necessary because Italian Law n. 447 had specifically requested that in evaluations and forecasts of long distance sound (greater than 250 meters), the influence of weather conditions on noise transmission should be considered. The evolution is significant if compared to the previous Guide de Bruit. Indeed, one proceeds from modeling based on abacuses to a more reliable description of road traffic seen in its complexity and incorporated into a spatial-temporal setting that is better at correctly representing the disturbance. The source of noise emissions is carried out by dividing the road or lanes into elementary point sources. With this subdivision, the receptor sees equal angles (generally $10^{\circ}$ ) between various source points. Alternatively, the elementary sources can be put at the same distance from each other (usually less than 20 meters). The calculation of the sound power level of an acoustic point source LAWi that represents a homogeneous road segment is given by:

$$
L_{W A i}=\left[\left(E_{V L}+10 \log Q_{V L}\right)+\left(E_{P L}+10 \log Q_{P L}\right)\right]+20+10 \log \left(I_{i}\right)+R(j),
$$


where:

- $E_{V L}$ and $E_{P L}$ are, respectively, the emission levels calculated with the abacus of C.ET.

UR. for light and heavy vehicles;

- Q $\mathrm{VL}_{\mathrm{V}}$ and $\mathrm{QPL}_{\mathrm{PL}}$ are the hourly flows of the same category of vehicles;

- $\mathrm{I}_{\mathrm{i}}$ is the length of a homogeneous segment of road;

- $R(j)$ is the value of the road noise spectrum drawn from EN 1793-3.

The introduction of the following information allows creating a complete model of road traffic: hourly flows of light and heavy vehicles; the speed of light and heavy vehicles; the type of traffic (continuous, throbbing, quick, slowed up); the number of lanes; the distance between the middle of the lane and the middle of the road; the features of the road section. In NMPB, the calculation of the propagation of sound is conducted for frequency bands whose center of band lies between 125 and $4000 \mathrm{~Hz}$. In 2001, the French standard XP S31-133 was published as an experimental norm. This is a methodology in which the basic measure describing sonorous input is the acoustic indicator already introduced in (2). Like the NMPB, the XP S31-133 standard describes a detailed procedure for calculating acoustic levels, taking into account weather effects (Licitra et al. 2012). Recently, on 19 May 2015, Directive 2015/996/CE was published; it established "Common noise assessment methods according to Directive 2002/49/EC of the European Parliament and of the Council" in order to standardize procedures and methodologies. However, the adjustment procedure for the new law is due in 2018.

Based on the results found in the literature (Licitra and Ascari, 2014), the $\mathrm{G}_{\text {den }}$ indicator was calculated for the same part of the historic center for which the noise map was developed. Also, the indicator was derived for other towns in Sicily and for other large population centers, so as to compare the results. The $\mathrm{G}_{\mathrm{den}}$ indicator, which has already been introduced for some time, was modified in the quoted study: "GDEN: an indicator for European noise maps comparison and to support action plans" in order to carry out a valuation of the results obtained in the acoustic maps drawn up in Europe in accordance with the END.

The new indicator, conventionally defined as normalized, is the sum of the exposition levels weighted by the number of people exposed:

$$
G_{\text {den norm }}=10 * \log _{10}\left(\frac{1}{N_{\text {tot }}} \Sigma_{i} n_{i} * 10^{\frac{L_{\text {d d }}}{10}}\right) \text {, }
$$

Where:

- $\mathrm{N}_{\text {tot }}$ is the total number of individuals exposed in the noise classes envisaged by END;

- $\mathrm{n}_{\mathrm{i}}$ is the number of people within each noise class;

- $\mathrm{L}_{\mathrm{deni}}$ is the central level of every exposition interval as defined by Directive 2002/49/CE.

The $\mathrm{G}_{\text {night }}$ indicator has been defined an analogous way. The initial data for determining these indicators were derived in part from the technical reports accompanying the acoustic mapping of the individual municipalities (these are freely available on the Internet) and in part from annuals of ISPRA (Istituto Superiore per la Protezione e la Ricerca Ambientale, The Italian National Institute for Environmental Protection and Research), that refer to the subject of noise. 


\section{MATERIALS AND METHODS}

\subsection{Analysis of cartography and creation of a DEM}

The Regional Technical Map (Carta Tecnica Regionale, CTR hereinafter) at scale 1:10000 and digital map at scale 1:2000 of urban centers (referred to as CTNC later in this discussion) have been used for this study. The CTR was available both in a computer-aided design (CAD) format (.dxf) and as shape files, while the CTNC only as shapefiles (a geospatial vector data format for GIS, .shp). The maps are in Gauss's projection with plane coordinates that refer to the Italian national system (Gauss-Boaga Rome 1940). Having at a precise and complex numerical map is essential when you are working in GIS (Costantino et al. 2016, Brigante and Radicioni, 2014). A first analysis evaluated whether the maps were suitable to the demands of the work. The first layers examined were those related to the morphology of the land. At both scales, this issue was fully treated, however, the presence of discontinuous polylines made problematic the creation of the DEM using the noise simulator software without first performing of geometric correction on the layers. Shapefiles were easier to use as input, indeed, each shapefile can be imported and, at the same time, allows one or more associations to be made between features present in its table and the corresponding features in the geofile created within the work situation. Once we had the morphology geofiles, it was possible to build the DEM, which was created on the basis of a Triangulated Irregular Network (TIN) model (Fig. 1).



Fig. 1 Triangulated Irregular Network (TIN) model for the study area.

\subsection{Integration of cartography with information on the resident population}

After building the DEM, heights of the residential areas were introduced to obtain a Digital Surface Model (DSM). Buildings, in addition to being an obstacle to the propagation of sound waves, physically represent the acoustic receptor within the model. The vectorization of buildings can be accomplished in two different ways with the noise simulator software. The first is based on the manual digitalization of every building, based 
on the geometric references of a vector file of the area concerned. Digitalization is less practical but safer in regard to the appropriateness and the completeness of data. For each building, it is possible to enter information such as the numbers of floors (stories), overall and individual story height and the number of residents. In such a highly-urbanized context (tens of thousands of buildings), this latter operation is time-consuming. The second method thus proved to be more suitable. This was a procedure that, as for the morphology, was based both on the import of a shapefile containing the buildings and on some connection associations between the features present in this vector file and those in the geofile of the noise simulator software. It was, however, necessary to examine the geometric congruence of the topological elements (gap, dangling, overshoots, intersections without nodes and duplicate lines). Since the DEM had already been created, the buildings, in addition to being planimetrically located, were placed accurately and automatically on the DEM. From considerations on the model of noise calculation and on the basis of data from the literature, it was decided, in the absence of data on the height of the building, to set a default mean value. This initial hypothesis, the consequences of which were then assessed, resulted in the modeling of conventional buildings with three floors and an overall height of 9 meters. To estimate the people exposed to the modeled sound levels, population count reported from Italian National Institute of Statistics (ISTAT, municipal population registers) were required. These data should ideally be geo-referenced to automatize the calculation procedure and for easy updating. The simplest procedure is based on the planimetrical intersection between curves of equal sound level and the census areas. In this procedure, resident inhabitants are directly proportional to the total area. Forecasting errors can be reduced by calculating the population density based on the buildings actual area alone. This was the procedure followed in this work. Where tridimensional digital cartographies were available, the buildings' height could be taken into account and volumetric population density could be used. In urban areas and for more detailed estimations, the use of the cartography containing house numbers is necessary. This cartography is now widely available in Italy. However, there are several difficulties, which restrict the use of these thematic maps. The determination of the exposed population is usually carried out by hypothesizing that all the people that live on a given street are exposed to the street's maximum noise level, not considering the presence of silent facades and how the individual flats are composed. In accordance with Directive 2002/49/CE (2017), the number of people that live in a building should be associated with the highest level of $\mathrm{L}_{\mathrm{den}}$ or $\mathrm{L}_{\mathrm{night}}$ of the most exposed facade, for every typology of noise. This estimate isn't easy to automatize and overestimates can be generated especially when the cartography of the buildings is poorly detailed (for example when several buildings are unified into a single block). In these cases, the opportunity to use different association criteria that give a more accurate estimate of the descriptor should be considered, even if they aren't completely exact. The desired final product was a geo-referenced raster map with the distribution of the population within the buildings' area. In light of the above and in anticipation of the valuation of the people exposed to the various noise bands generated by the acoustic map, correct data regarding the number and geographic localization of the population was needed. The available data, provided by the Municipality of Palermo, were found to be incomplete and fragmented, both as information in itself and as it was made available in digital format. These data originate from ISTAT's 2001 census and consist of: a polygonal shape file with the 2830 sections into which Palermo is divided and, for each of them, a record of the surface area and another for the perimeter; a .csv file containing, for each section, records of the surface area, the perimeter and an identification number (an 
identification number different from those in the shapefile); a second .csv file containing, for each section, an identification number (the same as the one in the .csv file) and data on the residents, which were divided into three categories - women, men and total. As a result of the fragmented nature of the data, to represent the spatial distribution of the value in question (the population) within the municipality, a new elaboration of available data using the open source GIS software was necessary. First, a new shapefile with the same attributes was derived from the shapefile of the census sections, but in such a way that it maintained the geo-referenced information. To that purpose, the centroids of every section were extrapolated, preserving the records of area and perimeter in the respective table of attributes and also adding two new records that indicated the East and North coordinates for every centroid (point). This was done because in the original file there was no information on the coordinates of every building. The file obtained made it possible to maintain correspondence between the area and the point object (Fig. 2).

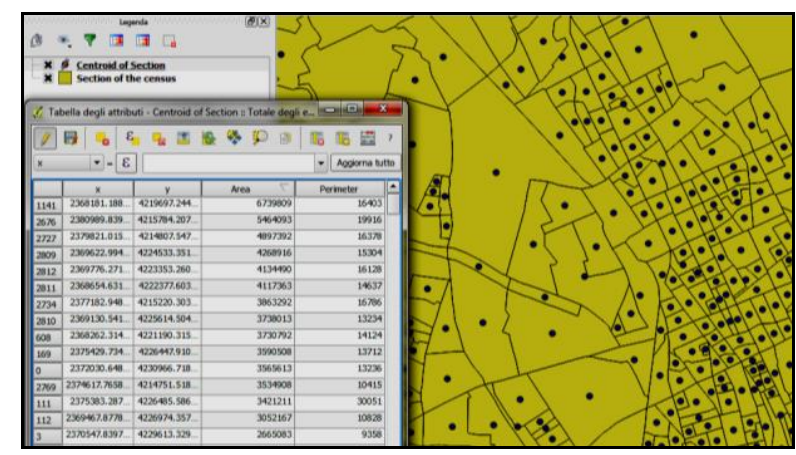

Fig. 2 Buildings centroids and attributes.

Afterward, the shapefile was exported in .csv and with the help of the spreadsheet, correspondences were created between the attributes of all the .csv files (two containing ISTAT data and one created during implementation). This allowed creating another spreadsheet listing, for every section of the census (identified with its own identification number), the surface area, the centroid's coordinates and the population residing there. A file with the number of people for every section but which considered these people as residing in the centroid was thus obtained. This seemingly simplistic assumption provided completely new information that was sufficiently accurate and credible for the job purpose. After carrying out a few operations with the GIS software tools, a new shapefile was created in which the population attribute was associated with the section, and not at its centroid created fictitiously. However, the data on the residents still presented an incongruity: inhabitants have been attributed to a single section without taking into account that each section contains areas suitable for building and others, which are not. The areas not suitable for the building were removed with other tools and new records with the population density (expressed in inhabitants per square meter) were created. The choice to calculate the datum per square meter and not per square kilometer was made based on the need to have a datum with a resolution similar to that of the acoustic map created afterward using a noise simulator software (SoundPLAN Acoustics, by SoundPLAN GmbH). In this way, map algebra tools could be used. Yet, since these operations are possible only for raster files, the shape files were first converted into raster format. To avoid losing precision, 
a resolution of one meter by one meter was maintained. This procedure, too, was made with the open source GIS software. The final product is visible in the underlying screenshot below that shows a part of the raster created (Fig. 3).

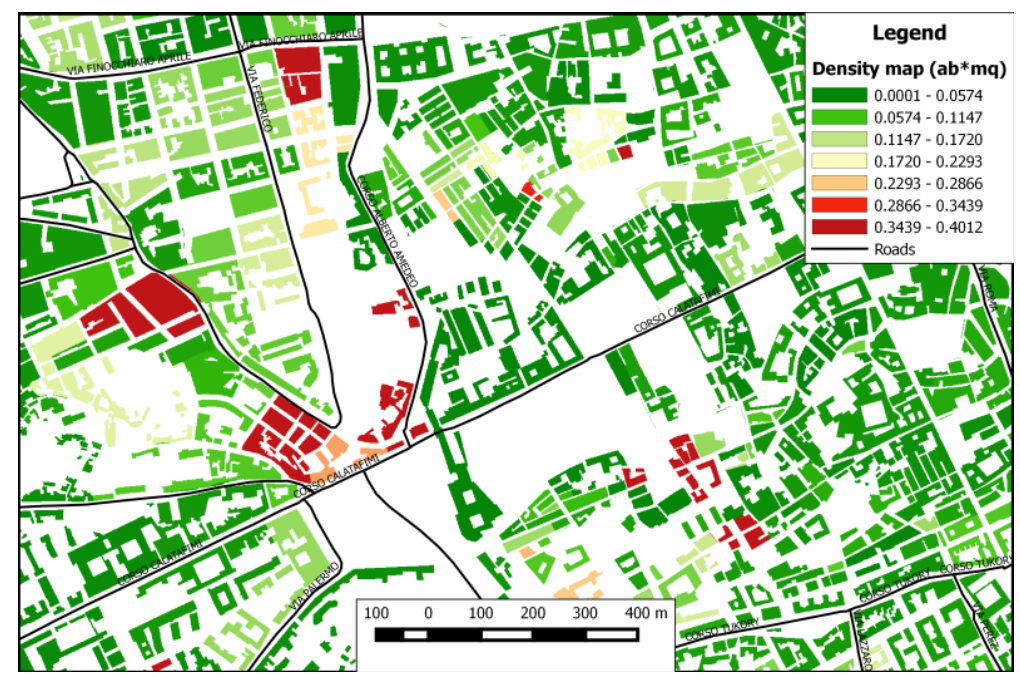

Fig. 3 Population density (detail).

As can be seen, the density varies substantially inside a single section. This is chiefly due to two reasons. The first is that the number of inhabitants is frequently divided among buildings nearby that are not residential buildings. The second, represented by the maximum density values, stems from the fact that buildings with many floors, and therefore with many inhabitants, have been modeled with only three floors, like the rest of buildings. The concentration is, therefore, higher in these buildings. Nevertheless, neither of these two factors was relevant for the purposes of the simulations that followed.

\subsection{Analysis, integration of traffic flows and creation of a database of noise sources}

After creating the DEM and after characterizing the receivers (the buildings with the inhabitants they contain), the following step was to import the noise sources into the noise simulator software. As for the other information levels, an initial analysis was carried out of the layers contained in the CAD file of the CTR and Regional Technical Numerical Map (Carta Tecnica Regionale Numerica, CTRN). It was found that the information of the streets was entirely or nearly nonexistent. The roads, or rather the street areas, are both implicitly displayed as the "empty spaces" of the other layers. The only information in these layers is the name of the streets themselves, but there is no information on their geometry. Here, too, although specifically dedicated layers were present, the information was highly fragmented and the attributes were not very homogeneous. This made it impossible to import the shapefiles as a street object into the software. Owing to these practical problems and bearing in mind that the traffic data (flows and speed) were necessary for each road, it was decided to create a new shapefile with all the information needed. In this way, as already seen with the other objects, it would have been easier to 
generate the street object into the noise simulator software with only the associations between the attributes. Before the vectorization of this shapefile, a study of the traffic data derived from the Urban Traffic Plan (UTP) was carried out, along with their analysis and subsequent integration. The UTP, amended in 2010, contains the report on the campaign for the measurement of vehicular traffic on the main roads. This campaign was made within the scope of the analysis of mobility that was realized for the General Urban Traffic Plan (GUTP, 2017) of the municipality of Palermo published in 2013. The survey of traffic flows concerned 85 streets (Fig. 4). Traffic flows were measured manually by teams of operators that recorded the data on relevant forms, taking care to classify this data into three morning time intervals (8:00 - 9:00; 9:00 - 10:00; 10:00 - 11:00 am). Vehicles were divided into five categories: cars; two wheel vehicles; commercial vehicles; buses; tourist buses. The campaign for measurement of traffic flows was made on carefully chosen days, in accordance with the literature. Saturdays, Sundays, (public) holidays and days with adverse weather events were avoided; specifically, the flows were measured on Tuesdays, Wednesdays, Thursdays and Fridays (Bastián-Monarca et al. 2016, Poggi, 2003, Google Earth, 2017, Pulighe et al. 2016).

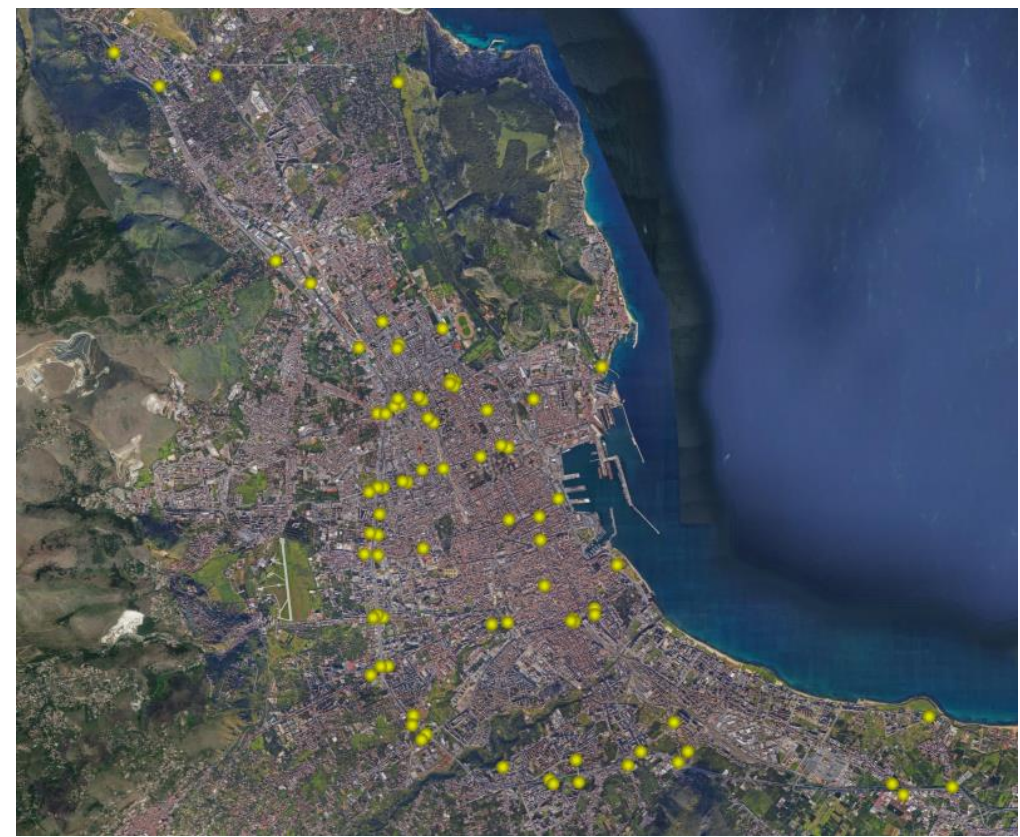

Fig. 4 Location of the measurement points (from Google Earth, approximate dimension $8 \mathrm{~km}$ x $5 \mathrm{~km})$.

A careful examination of the data included in the UTP made clear that although they describe a sufficient quantity of roads, they are too limited in time (from 08:00 to 11:00 am) and refer to just one day of the year; for this reason, the data aren't suitable for immediate implementation. In fact, the END gives precise rules on how to acquire and elaborate the traffic data for the purpose of determining the acoustic descriptors $\left(\mathrm{L}_{\mathrm{den}}, \mathrm{L}_{\mathrm{night}}\right)$. The Directive calls for a monitoring period of 24 hours, and to characterize the source of noise 
properly, the overall period measured must not be unduly influenced by temporary weather or environmental conditions. Assuming that the traffic data were representative for the 08:00 - 11:00 am interval, it was necessary to implement a procedure that extended the three - hour information into the three intervals called for by the Directive: daytime (06:00 - 20:00); evening (20:00 - 22:00); nighttime (22:00 - 06:00). As for the five categories of vehicles, these were reclassified in only two classes, as provided by current law: light vehicles (that included two-wheeled vehicles and cars), and heavy vehicles (that included commercial vehicles, buses and coaches).

The traffic data provided by ARPA Sicilia, obtained by the traffic counter devices in the same period as that of the UTP, were used to extend the data to 24 hours. Some of the same roads monitored for the UTP and by ARPA Sicilia were evaluated first; these roads differed in geometric type and in the number of transiting vehicles; they were also subject to different monitoring times (from a few days to two weeks). The roads were divided into two representative categories: "high vehicular flow roads" and "urban center roads". There was a weekly traffic dataset, but only weekday data were used; this dataset is more regular in the daytime period than on non-workdays. For each road category, three roads were selected as reference and for each one, the dataset was subdivided into two vehicle categories: "lightweight vehicles" and "heavy vehicles". For each road and vehicle category, the number of vehicles/hour was calculated and correlated to those in the period 10:00 - 11:00 a.m. (the traffic dataset for the UTP was in this period): the traffic data for every hour of the day were calculated from the 10:00 - 11:00 value using the "Transfer Coefficients" obtained. In the end, the average value of traffic data for each road and vehicle category was calculated. The time profile of the transfer coefficients varies greatly (Fig. 5) because it does not depend only on the number of vehicles per hour, but also on the urban area position and the time slot as well.

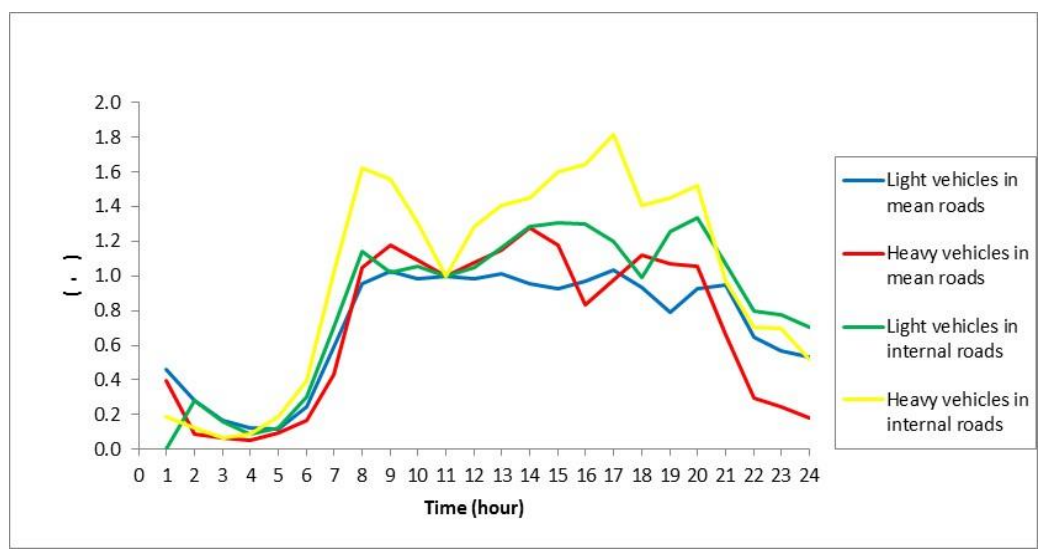

Fig.5 Transfer coefficients of traffic flow.

The methodology described is similar to the one used to calculate average hourly speeds; there was no speed dataset and this is a very important variable for implementation of the noise simulator software mathematic model.

Under the hypothesis of a similar distribution of traffic flow at various hours of the day, the number of vehicles and average hourly velocity for each road and vehicle category in the 24 one-hour slots of the day were calculated using transfer coefficients and the three- 
hour traffic dataset of the UTP. This hypothesis was verified by calculating the deviation between the 24-hour UTP dataset and our projection values from 8:00 - 9:00 a.m. (Fig. 6). For many of the roads, the final deviations at 9:00 a.m. were quite small (less than 15\%) or acceptable (between 30\% and 40\%); however, for a few roads, the deviations were greater due to mistakes made during the monitoring phases of the UTP (manual survey).

Most of the mistakes could be attributed to heavy vehicles due to their difficult classification; it is important to point out that the Noise Pressure Level increases by $3 \mathrm{~dB}$ if the number of Heavy vehicles doubles.

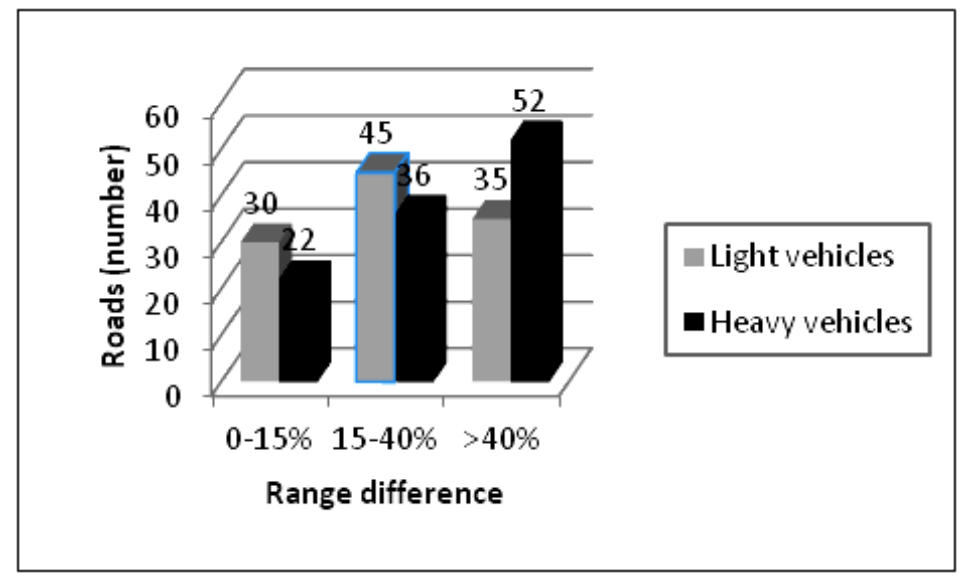

Fig. 6 Differences between actual and modeled values.

In conclusion, the monitored streets of the UTP were reclassified into two categories ("high vehicular flow roads" and "urban center roads") and the velocity and the traffic flow values were attributed to every street in each category. After the traffic dataset was obtained, the geometric properties (street weight, presence of a traffic divider, one-way or two-way traffic flow) were studied to position noise source lines on every street plan considered, then, a shapefile of street vectors was created with the open source GIS.

\subsection{Realization and analysis of acoustic maps}

Then the DEM was created and the shape files, the features of the buildings, the streets (noise sources) and the green areas (town parks, flowerbeds and permeable land areas) were uploaded into the "SoundPLAN Acoustic" software. Green areas absorb noise energy and produce a decrease in the Noise Pressure Level. The absorbent areas can be used in the noise simulator software modeling by introducing the parameter "Ground Factor". This parameter is the percentage of absorbed noise energy on an incident surface and a Ground Factor value of 0.6 was used for every green area. No acoustic reflecting boards were found in the town area. The methodology used to upload the absorbent features of green areas into the noise simulator software was the same one used to upload noise sources and building features: a shape file with the geometric features and a Ground Factor value were created for every absorbent area and it was uploaded into the noise simulator software. To start the simulation of noise wave propagation in the urban area, it was necessary to set the greatest number of wave reflections on building surfaces and the largest spatial range from noise 
sources so as to evaluate the spatial distribution of $\mathrm{L}_{\text {Aeq. }}$. With regard to the reflections, studies in the sector show that after the second reflection on a facade the level is greatly reduced, so the number two was chosen; however, in the second part of this study, the possible effect of a greater number of reflections was checked. As for the characteristics of the map, an output with a resolution of $3 \times 3$ meters was chosen. This choice increased the time required for calculations but gave a more detailed result that was suited for several subsequent analyses. Finally, we chose to represent the results with the European descriptors Lden and Lnight and not with Italian descriptors. Once the calculation run was completed, it was possible to see the results both as a schedule and as graphics with isolines. The first type of visualization is impractical and is good only for archiving data and for finding specific punctual values. Instead, a color map of the sound pressure level values proves to be more effective.

A very high level of sonorous pressure was found especially on the main roads constituting the axes of the city center (Fig. 7).

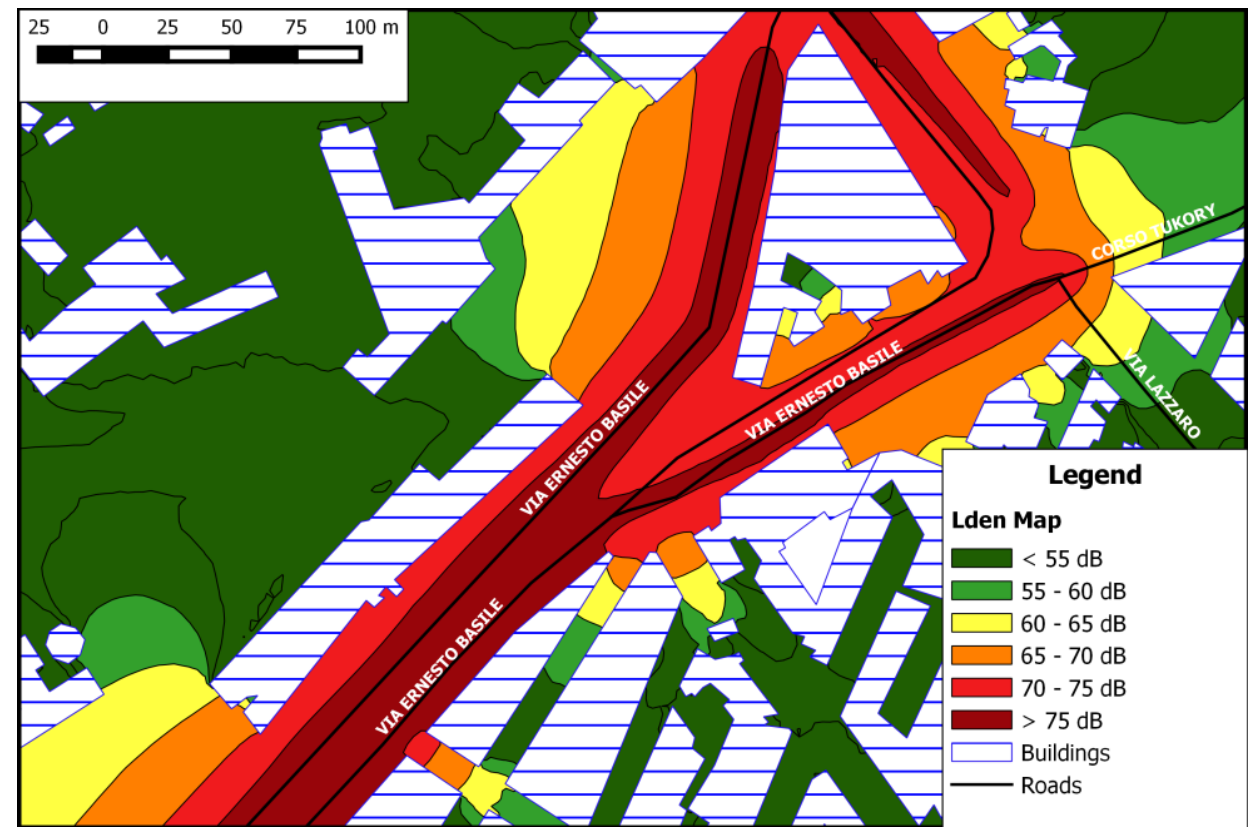

Fig. 7. Particular of Lden map (e.g. Ernesto Basile road).

The map relates to the area around the university and the residences of Ernesto Basile road. As can be seen, noise level exceeds $70-75 \mathrm{~dB}$ in some areas. Similar situations also occur in other parts of the city. Once we had the shapefiles of the noise maps and the raster relating to the population density, sound pressure levels of the exposed population were evaluated. This analysis was carried out with the help of the open source GIS. To obtain the correct data, at first the Geoprocessing instruments for the vector file were used; once this was done, we imported the raster of the population density for every square meter of built surface area and with the help of the tools, the following zonal statistics were obtained for each range of $5 \mathrm{~dB}$ : the number of pixels relating to the buildings contained in each range (in this case, since the pixels were equivalent to one square meter, the information matched 
that relating to the built surface contained in each range); the sum of the values of the pixels that fall inside each range (this information coincided with the number of inhabitants for the entire noise interval); an average density value for each interval (expressed in inhabitants for square meter). Once the data from the zonal statistics had been collected and processed, it was easy to determine how the population was distributed within the noise maps $\mathrm{L}_{\mathrm{den}}$ and $\mathrm{L}_{\text {night. }}$ First, it should be noted that compared with about 680,000 inhabitants of Palermo, those contemplated in the simulation numbered just over 89,000 (the population relating to the 85 roads involved in the simulation). The study revealed, in line with other studies conducted at the national and European Community level, an unflattering scenario indeed, an alarming one. As can be observed in the following histograms (Fig. 8), with regard to Lden level, there is about a third of the investigated population that lives with a sound pressure level of less than $55 \mathrm{~dB}$, while, on the other hand, it is remarkable that $29 \%$ of the population is forced to live with values that exceed $65 \mathrm{~dB}$ throughout the day, and as much as $7 \%$ is subject to levels higher than $75 \mathrm{~dB}$.

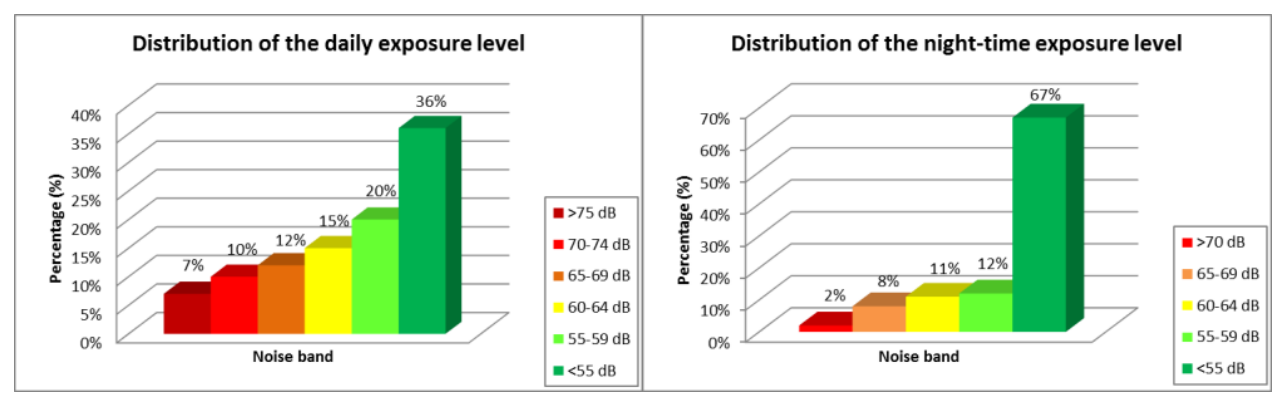

Fig. 8 Distribution of the day-evening-night time exposure level (left) and nighttime exposure level (right).

At night, though there is a general and physiological attenuation, levels often remain high and are unfit for proper rest. In fact, about $67 \%$ of the investigated population is subjected to a sound pressure $\leq 55 \mathrm{~dB}$, while one in three people is subjected to pressure that exceeds that level and there is also $\approx 10 \%$ that at night must bear levels of traffic noise $>65 \mathrm{~dB}$.

\section{RESULTS AND DISCUSSION}

The area under investigation is a part of the historic center of Palermo (Fig. 9), and this area includes nearly the entire first district according to the current municipal division scheme. In the simulations carried out previously, a building height of 9 meters was established and only the first two reflections were considered. In the second part of the job, we considered the differences in the results produced by two additional calculations, in which:

- $\quad$ the height of the buildings was $9 \mathrm{~m}$ and the number of reflections was 5 (changing only the number of reflections);

- $\quad$ the height of the buildings was $18 \mathrm{~m}$ and the number of reflections 5 (unchanged). 


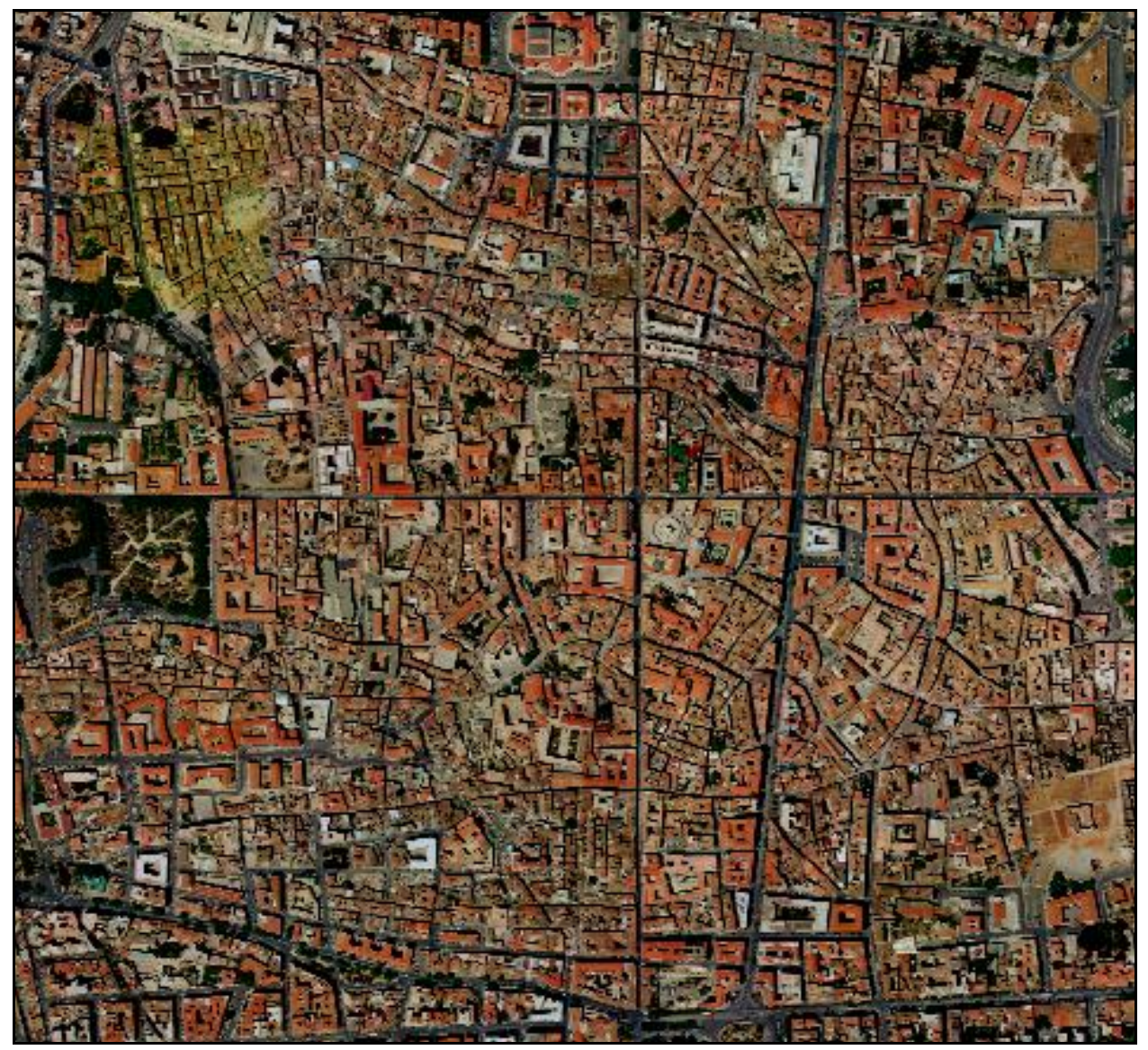

Fig. 9 Simulation area: historic center of Palermo (approximate dimension $1 \mathrm{~km}$ x $1 \mathrm{~km}$ ).

As evidenced in the following pictures (Fig. 10), the influence of one or both parameters considered determined at most a shift into the next range, and therefore an increase of $5 \mathrm{~dB}$. This value is not negligible, but by looking at the percentage of pixels involved in this change (and thus the amount of surface area), we can safely affirm that when the variation of these two parameters is so limited, it is irrelevant for the final product. In any case, in regard to the two parameters, the greater impact was caused by increasing the number of reflections from 2 to 5 , which determined a variation of about 12 $\%$ in the number of pixels in regard to the daytime map. This value decreased in the nighttime map where it was slightly higher than $2 \%$.

On the other hand, in the maps in which the height of buildings changed from 9 to 18 $\mathrm{m}$, there were no significant variations (only a few points per thousand) compared to the maps where only the number of reflections was increased and not the number of floors. It can be seen in the nighttime maps that the variations are even less obvious in comparison with the daytime map, maybe because the source of noise is less intense and is dispersed in a smaller space and thus is less subject to effects of amplification or mitigation. 


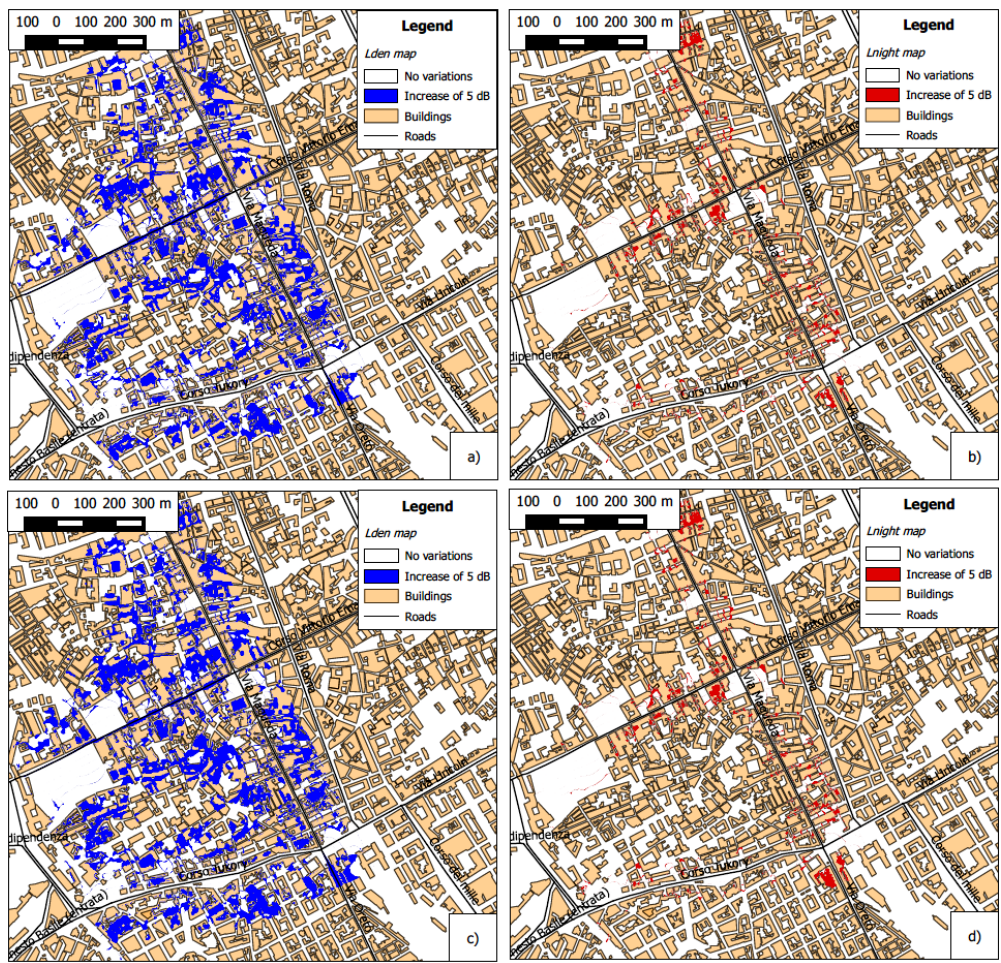

Fig. 10 Comparison between the initial simulation and the following simulations a) $\mathrm{L}_{\mathrm{den}}$, changing number of reflections; b) $\mathrm{L}_{\text {night }}$, changing number of reflections; c) $\mathrm{L}_{\mathrm{den}}$, varying number of reflections and height of buidings; d) Lnight, varying number of reflections and height of buildings.

Table 1. Inhabitants within each range of acoustic pressure (in thousands).

\begin{tabular}{|c|c|c|c|c|c|c|c|c|c|c|c|c|}
\hline Town & \multicolumn{4}{|c|}{$L_{\text {den }}$ range (db) } & $\begin{array}{c}\text { Tot. } \\
\text { den }\end{array}$ & \multicolumn{5}{c|}{$L_{\text {night range (db) }}$} & $\begin{array}{c}\text { Tot. } \\
\text { night }\end{array}$ \\
\hline & $\mathbf{5 5 - 5 9}$ & $\mathbf{6 0 - 6 4}$ & $\mathbf{6 5 - 6 9}$ & $\mathbf{7 0 - 7 4}$ & $\mathbf{> 7 5}$ & & $\mathbf{5 0 - 5 4}$ & $\mathbf{5 5 - 5 9}$ & $\mathbf{6 0 - 6 4}$ & $\mathbf{6 5 - 6 9}$ & $>\mathbf{7 0}$ & \\
\hline Cagliari & 12 & 26 & 60 & 52 & 7 & 157 & 21 & 45 & 69 & 14 & 2 & 151 \\
\hline Bologna* & 87 & 86 & 83 & 67 & 16 & 339 & 76 & 84 & 59 & 29 & 1 & 249 \\
\hline Turin & 36 & 375 & 208 & 214 & 28 & 861 & 241 & 272 & 197 & 126 & 6 & 842 \\
\hline Florence & 99 & 71 & 92 & 42 & 2 & 305 & 82 & 93 & 52 & 5 & 0 & 231 \\
\hline Milan & 183 & 273 & 254 & 223 & 51 & 984 & 271 & 268 & 247 & 70 & 1 & 856 \\
\hline Genova & 62 & 82 & 41 & 14 & 4 & 203 & 80 & 70 & 24 & 7 & 1 & 183 \\
\hline Parma & 77 & 81 & 19 & 1 & 0 & 178 & 119 & 46 & 5 & 0 & 0 & 169 \\
\hline Rome*** & 1845 & 341 & 72 & 58 & 5 & 2320 & 323 & 67 & 57 & 7 & 2 & 456 \\
\hline Bari & 51 & 98 & 68 & 29 & 0 & 247 & 89 & 68 & 50 & 6 & 0 & 213 \\
\hline Palermo** & 18 & 13 & 10 & 9 & 6 & 56 & 15 & 11 & 10 & 8 & 2 & 44 \\
\hline
\end{tabular}


* (Data refer to the entire conurbation of Bologna)

** (Data on the population affected in daytime differ from the one during nighttime)

*** (Investigated population does not match with the total number of inhabitants)

Finally, with regard to the spatial distribution of the pixels subjected to these variations, pixels do not appear to follow any particular trend. In the table above (Table 1) the data related to the cities studied are summarized, in which $n_{i}$ (the number of people inside the single noise class) is in thousands. Urban areas as Turin, Milan and Rome are characterized by a relatively high number of inhabitants within each range of acoustic pressure (dark grey), while, viceversa, although Palermo reaches each range of acoustic pressure with the lowest number of inhabitants.

The input data was elaborated and yielded the results summarized (Fig. 11).

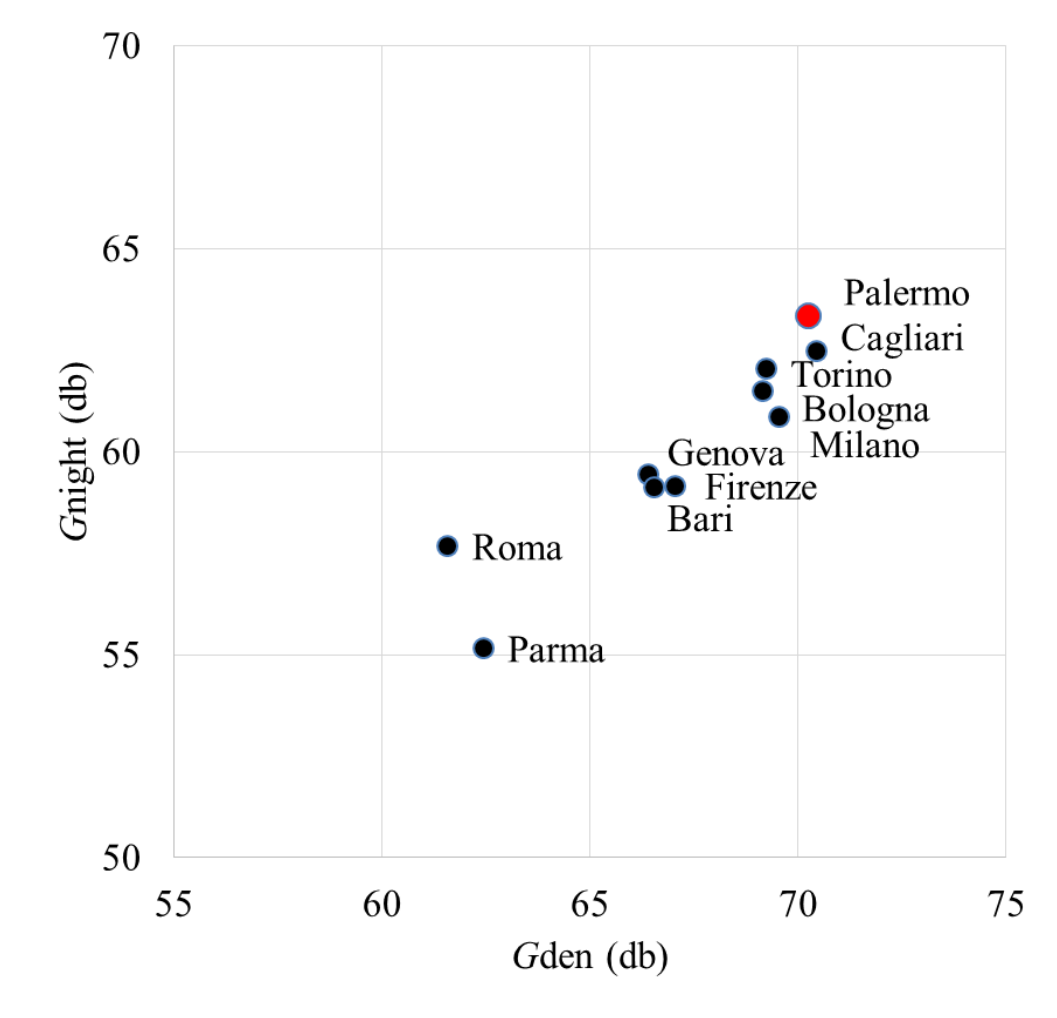

Fig. 11 Comparison between $G_{\text {den }}$ (x axis), $G_{\text {night }}$ (y axis) indicators of the principal Italian urban areas.

Indicators show that Palermo ranks among the noisiest towns in the national context $\left(\mathrm{G}_{\mathrm{den}}=70.2 \mathrm{~dB}, \mathrm{G}_{\text {night }}=63.4 \mathrm{~dB}\right)$. These values, however, do not differ significantly from average values of the test sample, which are respectively 67.2 and $59.7 \mathrm{~dB}$. 
It is, nevertheless, important to emphasize that the investigated population lives in areas where traffic noise is the highest. For this reason, it is presumed that if indicators were re-calculated when the noise map is complete, these would decrease by some units close to those of the more virtuous towns.

\section{CONCLUSIONS}

This study realized the first acoustic map for vehicular traffic in the urban center of the city of Palermo. It was evident in the first analysis of the traffic data provided by the Urban Traffic Plan that the general situation of the town was rather congested. In this context, a heterogeneous situation was found. The first clear difference was found in the distribution of traffic flows. Three distinct scenarios could be identified: main roads for access to the city, on which there are very steady daily trends and considerable traffic flows distributed on wide streets; roads of the city center where there are heavy traffic flows, mainly consisting of traffic to and from work and school, but on rather narrow carriageways; roads of peripheral areas whose flows are not significant in the economy of the macro-area.

As already mentioned in the Results and discussion section, it is important to stress that though there are 680,000 inhabitants in the Palermo city center, those interested by the simulation were just about 89,000 . This datum reflects the number of inhabitants that live in the buildings next to the 85 roads for which traffic data had been obtained. With regard to the roads where no traffic measurements have been made, it was decided not to use default values, as it is often assumed, and, at the same time, not to attribute a value using a criterion of similarity with other roads. Although this choice reduced the number of people involved in the simulation, it produces a closer correspondence to the actual situation. The study of the people involved within each band of noise revealed, in line with other national and European studies, an unflattering scenario.

With regard to the day-evening-night map Lden, it was found that about one-third of the investigated population is subject to a sound pressure level lower than $55 \mathrm{~dB}$, a value suitable for conducting all daytime activities. About three people out of ten (29\%) co-exist with daytime values above $65 \mathrm{~dB}$, while there is $7 \%$ that tolerate sound levels $>75 \mathrm{~dB}$ through the whole day. At night, even though there is a general and obvious attenuation, the levels often remain high and unsuitable for sleeping. Though $67 \%$ of the people live with a sound pressure value $\leq 55 \mathrm{~dB}$, one person in three is subjected to a value $>55 \mathrm{~dB}$. There is also $\approx 10 \%$ of the population in the investigated areas that in the nighttime hours is subjected to a sound pressure level $>65 \mathrm{~dB}$, values which greatly exceed the limits prescribed by national laws.

With reference to the Gden method, in comparison with other Italian cities, it was found once again that Palermo is among the cities where the population is subjected to the greatest impact. The Gden and Gnight data, though they do not differ greatly from the national average, indicate very high noise levels. As already underlined in the discussion, the data for the internal construction of the indicators (Weber, 2011) are strongly dependent on the number of people in the highest noise classes. It is believed that in this yearly mapping of Palermo, information pertaining to the most critical cases was given, not taking into consideration less noisy roads, which, in a global vision, would diminish the overall effects of traffic noise (Wei et al. 2014). 
When the mapping for the entire town has been completed, the use of the indicators $\mathrm{G}_{\text {den }}$ and $\mathrm{G}_{\text {night }}$ would be a good place to start for the analysis of certain small-scale situations (census sections, blocks, districts) and to establish priorities for interventions prescribed by action plans. It became clear during the performance of the work that in general, the greatest difficulties encountered in the elaboration of the simulation were due to the weaknesses in the cartography and the information and data on the noise sources considered in the specific situation.

More specifically, these must be complete and appropriate for use with the software and must allow three-dimensional models to be accurately created in the study context. Indeed, it was noted that the available cartography is inadequate for the requirements of acoustic mapping for several reasons.

Among the main inadequacies, it does not include buildings as 3D objects, (in fact there is no information on the number of floors or on buildings' height) and there is no complete information on the roads, whose geometric characteristics should be defined so that software can easily represent them. Furthermore, especially for the roads, the characteristics of the pavement should be defined for every typology because they represent a factor that influences the noise contribution of tires and the attenuation of noise. Moreover, the necessary data on traffic flows and average speeds, which should be separated according to typology (light or heavy), and every time slot envisaged by European and Italian laws on acoustic pollution should be assessed.

It is also important to have easy access to the data on the distribution of people in the urban territory, not only in the section but also in every building. This would facilitate the evaluation of the population involved, not only with regard to the problem of noise but to every environmental issue, without the need to implement, as in this study, a complex procedure that nevertheless leaves open margins for error.

Also examined was the influence of certain choices regarding the geometric configuration of the buildings examined and certain physical parameters crucial for calculations. In this respect, it was noted by examining a reduced area of the historic city center that the differences with the maps were very limited, and only a few small areas entered into the higher acoustic band. More specifically, the prime factor contributing to this shift was the hypothetical increase in the number of reflections in the daytime map, which produced a variation in about $12.5 \%$ of the surface involved. This value decreased to just over $2 \%$ in the nighttime map.

The number of reflections appears influenced by the distance between the facing buildings; for this reason, in urban canyons (with reduced width of the roadway) can take into account the successive reflections at the second. With regard to the variation due to the number of floors, this amounted to a few points per thousand and was therefore completely irrelevant in the context.

Finally, it seems correct to invoke more dynamic consultation and updating of environmental information without the constraints and limits imposed today by fragmentation of the various tasks. In this context, an open source data policy and the online sharing of resources would help reach targets that now seem distant, while simultaneously satisfying all legal obligations. 


\section{ACKNOWLEDGMENT}

This work was developed during the training and thesis stage of a post-graduate Master's Program entitled "Research Technician Specialized in the Determination and Management of Environmental Risk through the use of ICT Network Solutions"; it was financed by the Interuniversity Consortium for Physics of the Atmospheres and the Hydrospheres (CINFAI) and it was conducted in collaboration with the DICAM of the University of Palermo and the Laboratory of Physical Agents of the Regional Agency for the Protection of the Environment (ARPA Sicilia).

\section{R E F E R E N C E S}

Directive 2002/49/EC of the European Parliament and of the Council of 25 June 2002 (2017). [Online] Available from http://eur-lex.europa.eu/eli/dir/2002/49/oj [Accessed Jun 2017].

GUTP (General Urban Traffic Plan) (2017). [Online] Available from https://www.comune.palermo.it/js/server/uploads/trasparenza_all/_17042014100310.pdf[Access ed August 2017].

Google Earth (2017) Google Earth Image. [Online] Available from www.google.com/earth/[Accessed August 2017].

Sicily Region Office (2017). [Online] Available from www.regione.sicilia.it/turismo/trasporti/CE/port\%20net/fin\%20IAE\%20nI/fin\%20L\%204471995.pdf. [Accessed August 2017].

Bastián-Monarca, N.A., Suárez, E., Arenas, J.P. (2016) Assessment of methods for simplified traffic noise mapping of small cities: Casework of the city of Valdivia, Chile. Science of the Total Environment, 550, pp. 439-448.

Costantino, D., Angelini, M.G., Claveri, M., \& Alfio, V.S. (2016) Document GIS and DBMS implementation for the development of rural areas of the "One hundred masserie" of Crispiano.Geographia Technica, 11 (1), 23-32.

Prekop, M., Dolejš, M. (2016) Do bypass routes reduce noise disturbances in cities? Case study of Cheb (Western Bohemia, Czech Republic).Geographia Technica, Vol 11, Issue no. 2/2016, pp. 78-86, DOI: 10.21163/GT_2016.112.08

Pulighe, G., Baiocchi, V., Lupia, F. (2016) Horizontal accuracy assessment of very high resolution Google Earth images in the city of Rome, Italy.International Journal of Digital Earth, 9 (4), 342362, doi: 10.1080/17538947.2015.1031716.

Cai, M., Zou, J., Xie, J., Ma, X (2015) Road traffic noise mapping in Guangzhou using GIS and GPS. Applied Acoustics, 87 pp. 94-102.

Brigante, R., \& Radicioni, F. (2014) Georeferencing of historical maps: Gis technology for urban analysis. Geographia Technica, 1, 2014, 10-19.

Licitra G., Ascari E. (2014) Gden: An indicator for European noise maps comparison and to support action plans. Science of The Total Environment - Volumes 482-483, pp. 411-419.

Wei W., Botteldooren D., Renterghem T.V., Hornikx M., Forssén J., Salomons E., Ögren M. (2014) Urban background noise mapping: The general model. Acta Acustica united with Acustica, 100 (6), pp. 1098-1111.

Curcuruto, S., Silvaggio, R., Amodio, R., De Rinaldis, L., Mazzocchi, E., Sacchetti, F., Stortini, M. (2012) HUSH project contribution to environmental noise directive implementation and revision, focusing on noise management and public information tools. 41st International Congress and Exposition on Noise Control Engineering, INTER-NOISE, 5, pp. 4036-4045.

Licitra, G., Ascari, E., Brambilla, G. (2012) Comparative analysis of methods to estimate urban noise exposure of inhabitants. Acta Acustica united with Acustica, 98 (4), pp. 659-666. 
Seong, J.C., Park, T.H., Ko, J.H., Chang, S.I., Kim, M., Holt, J.B., Mehdi, M.R. (2011) Modeling of road traffic noise and estimated human exposure in Fulton County, Georgia, USA. Environment International, 37 (8), pp. 1336-1341.

Weber, M. (2011) A new area-based noise indicator Gden: Assessing noise annoyance in residential areas. 40th International Congress and Exposition on Noise Control Engineering, INTER-NOISE 4, pp. 3428-3434.

Covaciu, D., Cofaru, C., Florea, D., Timar J. (2010) Aspects regarding the road traffic noise and its effect on the population. International Conference on Urban Planning and Transportation Proceedings 2010, pp. 86-91.

Dardanelli G., Sansone Santamaria A., Marretta R., Calà Lesina N. (2010) Application of Directive END for the acoustic mapping by vehicular traffic. The case study of historic center of Palermo. Proceeding of XIV National Conference ASITA 2010, pp. 751-756 (In Italian with English Abstract).

Murphy, E., King, E.A. (2010) Strategic environmental noise mapping: Methodological issues concerning the implementation of the EU Environmental Noise Directive and their policy implications. Environment International, 36, pp.290-298

O’Malley, V., King, E., Kenny, L., Dilworth, C. (2009) Assessing methodologies for calculating road traffic noise levels in Ireland - Converting CRTN indicators to the EU indicators (Lden, Lnight). Applied Acoustics, 70 (2), pp. 284-296.

Hepworth, P. (2006) Accuracy implications of computerized noise predictions for environmental noise mapping. Institute of Noise Control Engineering of the USA - 35th International Congress and Exposition on Noise Control Engineering, INTER-NOISE, 6, pp. 3586-3591.

Poggi, A. (2003) Noise pollution measurement procedures: A comparison between Italian legislation and EU 02/49 directive. Acta Acustica (Stuttgart), 89 (SUPP.), pp. S91.

Knauss, D. (2002) Noise mapping and annoyance. Noise and Health, 4 (15), pp. 7-11.

CERTU-CSTB-LCPC-SETRA (1997). Bruit des infrastructures routières - Méthode de calcul incluant les effets météorologiques (Nouvelle Méthode de Prévision du Bruit -NMPB-Routes1996), Paris, France. 